\title{
A genetic model for multimorbidity in young adults
}

\author{
Sarah L. Malecki, MD ${ }^{1,2}$, Spencer Van Mil, MD², Justin Graffi, MSc ${ }^{2}$, Elemi Breetvelt, MD, PhD ${ }^{3}$, \\ Maria Corral, MD, FRCPC ${ }^{3,4}$, Erik Boot, MD, PhD ${ }^{3,5,6,7}$, Eva W. C. Chow, MD, FRCPC 2,4 \\ Marcos Sanches, MSC ${ }^{8}$, Amol A. Verma, MD, FRCPC ${ }^{9}$ and Anne S. Bassett, MD, FRCPC ${ }^{2,3,4,10,11}$
}

Purpose: Multimorbidity is increasing in younger adults but is understudied in this population. We used 22q11.2 deletion syndrome (22q11.2DS) as a genetic model to investigate multimorbidity in young to middle-aged adults.

Methods: Using the Anatomical Therapeutic Chemical (ATC) Classification System and setting five or more concurrent prescription medications as a proxy for multimorbidity, we compared data on 264 adults with 22q11.2DS (median age 27.8, range 17.3-68.3 years) with that for a community-based Canadian general population sample $(n=25,287)$. We used logistic regression to examine possible predictors of multimorbidity in 22q11.2DS.

Results: Multimorbidity in 22q11.2DS in the 25-44 year age group $(34.7 \%)$ was significantly more prevalent than in the general population, both for the same age group $(2.9 \%$, prevalence ratio $[\mathrm{PR}]=11.9,95 \% \mathrm{CI}$ 8.4-17.1) and compared with those aged 45-64 years $(16.4 \%, P R=2.1,95 \%$ CI $1.6-2.7)$. Neuropsychiatric and endocrinological medication classes predominated. Within 22q11.2DS, older age and psychotic illness, but not sex, major congenital heart disease, or intellectual disability, were significant predictors of multimorbidity.

Conclusion: The results indicate that adults with 22q11.2DS have a significant burden of illness with levels of multimorbidity comparable with those of the general population several decades older. In younger adults with multimorbidity, certain disease patterns may help identify genetic disorders in "big data."

Genetics in Medicine (2020) 22:132-141; https://doi.org/10.1038/s41436019-0603-1

Keywords: noncommunicable diseases; drug therapy; multiple chronic conditions; rare genetic disorders; DiGeorge syndrome

\section{INTRODUCTION}

Multimorbidity, or the concurrence of two or more medical or psychiatric conditions, ${ }^{1}$ increases with age and is related to higher health-care spending and adverse outcomes including mortality. ${ }^{2-4}$ Geriatric populations are well studied but less is known about the etiology of the increasing multimorbidity observed in younger adults ${ }^{5-7}$ where patterns may differ from those of the elderly. ${ }^{3}$

Possible explanations for increasing multimorbidity in young adults ${ }^{8}$ include lifestyle changes resulting in more cardiometabolic disorders, and increasing prevalence of mental health conditions like anxiety and depression. A potentially unrecognized explanation is the improved survival of children with genetic conditions due in part to advances in pediatric care over the past 50-60 years. Included in this growing number of adults are those with rare pathogenic copy-number variations $(\mathrm{CNVs})$, many recurrent, that may predispose to multisystem illness. ${ }^{9-13}$ Collectively, individual rare diseases such as these are common, estimated to affect about $5 \%$ of the population in developed countries. ${ }^{14}$ Many have substantial burden of illness yet without obvious dysmorphic features or intellectual disability (ID). This includes the most common microdeletion syndrome in humans, 22q11.2 deletion syndrome (22q11.2DS), present in an estimated 1 in 3000 live births. ${ }^{13}$ The syndrome has variable presentation and is associated with congenital and later onset problems, including cardiac malformations, hypoparathyroidism, hypothyroidism, cognitive delays, and neuropsychiatric disorders, and with premature mortality. ${ }^{12,13,15}$ Clinical samples are currently the only means for studying this and many other genetic conditions due to lack of coding and algorithms for identification in large administrative databases. ${ }^{16-18}$

Using 22q11.2DS as a model genetic disorder, we hypothesized that multimorbidity in young to middle-aged

\footnotetext{
${ }^{1}$ Internal Medicine Residency Program, University of Toronto, Toronto, ON, Canada; ${ }^{2}$ Clinical Genetics Research Program, Centre for Addiction and Mental Health, Toronto, ON, Canada; ${ }^{3}$ The Dalglish Family 22q Clinic, University Health Network, Toronto, ON, Canada; ${ }^{4}$ Department of Psychiatry, University of Toronto, Toronto, ON, Canada; ${ }^{5}$ Heeren Loo Zorggroep, Amersfoort, The Netherlands; ${ }^{6}$ Department of Nuclear Medicine, Academic Medical Center, Amsterdam, The Netherlands; ${ }^{7}$ Department of Psychiatry \& Neuropsychology, Maastricht University, Maastricht, The Netherlands; ${ }^{8}$ Biostatistical Consulting Service, Centre for Addiction and Mental Health, Toronto, ON, Canada; ${ }^{9}$ Li Ka Shing Knowledge Institute \& Department of Medicine, St Michael's Hospital, University of Toronto, Toronto, ON, Canada; ${ }^{10}$ Campbell Family Mental Health Research Institute, Toronto, ON, Canada; ${ }^{11}$ Division of Cardiology \& Toronto General Hospital Research Institute, University Health Network, Toronto, ON, Canada. Correspondence: Anne S. Bassett (anne.bassett@utoronto.ca)
} 
adults with 22q11.2DS would be greater than that in a large community-based general population sample. Secondary aims were to investigate the pattern of, and identify main contributors to, multimorbidity in this genetic model.

\section{MATERIALS AND METHODS}

\section{Study design and setting}

This was a cohort study based on review of medications coded from extensive medical records in an outpatient sample of adults with 22q11.2DS and comparably coded medication data available for a large community-based general Canadian population sample. The study was approved by the local research ethics boards of hospitals (Centre for Addiction and Mental Health and University Health Network) affiliated with the University of Toronto.

\section{Proxy for multimorbidity}

We chose prescription medication use as a proxy measurement for multimorbidity. There is no consensus on the best way to measure multimorbidity, ${ }^{1}$ but previous work suggests a simple count of prescription medications may be a reasonable proxy when studying community samples. ${ }^{19}$ This proxy has several benefits over existing multimorbidity indices that were developed for use in older adults, ${ }^{20}$ exclude potentially important conditions, ${ }^{21}$ and rely on recording of diseases, thus may not be reliable or standardized for use among different data sets. ${ }^{1}$ Also, medication use often indicates a more severe, and perhaps more significant, form of disease. ${ }^{22}$ We chose a conservative threshold of five or more concurrent prescription medications, used previously to define multimorbidity. ${ }^{23}$ While a commonly used threshold is two or more conditions, more stringent definitions have greater specificity. $^{24}$

\section{Data sources}

\section{2q11.2DS data}

The 22q11.2DS sample comprised 264 individuals (140 females, 53\%, median age 27.8, range 17.3-68.3 years) from a well-characterized outpatient cohort $^{12,25}$ where sufficient adult data (collected at $\geq 17$ years of age) were available on medications, and a typical 22q11.2 deletion was confirmed by standard molecular methods. ${ }^{12,13,15}$ Most subjects were ascertained through screening at an adult congenital cardiac clinic, and/or through genetic or psychiatric referrals, as previously described. ${ }^{25}$ Informed consent was obtained for all individuals in the study. We reviewed medical records for the entire 22q11.2DS cohort enrolled as of July 2016.

We recorded information on concurrent, regular medication use at one specific time point, using the most recent information available to record the date of clinical assessment and each medication taken at that time. Most data were recorded from a detailed clinical summary for each subject, updated at annual visits to a 22q11.2DS specialty clinic in Toronto, and based on a combination of information gleaned from reviewing medical and patient pharmacy records, patient self-report, and review of pill bottles brought to clinic.
In the minority of cases where a clinical summary was not available, the most recent medical and/or pharmacy records were used. Review of medical records proceeded from July 2016 to June 2017, based on medical records obtained up to December 2016. The median year at medication recording was 2015 (Q1 2013, Q3 2016). Age at medication use was calculated based on date at medication recording and rounded to the nearest 10th of a year. For one subject where only the year of medication use was known, the date assumed was 15 June.

We included all routes of administration for prescription medications and defined regular use as daily, weekly, monthly, or up to every 3 months for injectables such as ustekinumab, Depo-Provera, and intravenous immune globulin. We defined medications as "prescription" based on Canada's Prescription Drug List. $^{26}$ Prescription vitamin preparations of calcitriol and alfacalcidiol were included. Nasal steroids and topical skin treatments, including steroids, antifungals and antibiotics, as well as lactulose regardless of dose, were assumed to be prescription. Other medications not included on Canada's Prescription Drug List were assumed to be over-the-counter medications and were thus excluded. These included acetaminophen, nonsteroidal antiinflammatory drugs, aspirin, benzoyl peroxide, loperimide, senna, polyethylene glycol, vitamins/supplements, and probiotics, herbal, naturopathic, and homeopathic remedies. We therefore excluded the only prophylactic treatments (vitamin $\mathrm{D}$, calcium supplementation) recommended for $22 \mathrm{q} 11.2 \mathrm{DS} .^{27}$ In accordance with our definition of regular use, we excluded short-term treatments recommended before dental surgery or a plane trip, and annual influenza vaccinations. Since there was no way to determine how often they were being taken, we excluded all medications noted to be taken as needed (PRN). We classified medications based on the Anatomical Therapeutic Chemical (ATC) Classification System. $^{28}$ Each medication recorded was assigned an ATC code with as many digits as possible (up to seven) given the level of detail available for the medication.

\section{Canadian population data}

We gained access to Canadian population data on prescription medication use through the Research Data Centres (RDC) data access program at Statistics Canada. Data were derived from the combined Canadian Health Measures Survey (CHMS) cycles 1-4 (2007-2015) (total $N=25,287$; median age 40; Q1 24, Q3 55 years; $50.1 \%$ female). This is a national survey sampling the Canadian population aged 3-79 years. This survey comprises a primary household visit and secondary clinic component, with information on risk indicators, disease conditions, and medications collected. Applying bootstrap weights to the survey data enabled derivation of estimates representative of the Canadian population. To improve reliability over self-report, information about prescription medications taken in the last month was collected using substantial objective data (i.e., visiting the home of participants and asking them to retrieve their pill 
bottles). For more details on the CHMS data, see Supplemental Methods.

\section{Data analysis}

For both the 22q11.2DS and population ATC-coded data, we tabulated the number of prescription medications per person. For combining and tabulating CHMS population data, analyses were conducted using Stata version 15 (StataCorp LLC, College Station, TX) and/or SPSS version 25 (IBM Corp., Armonk, NY). We calculated prevalence estimates and $95 \%$ confidence intervals (CIs). Survey weights were used to obtain population estimates and 500 bootstrap weights provided with the surveys were used to estimate standard errors and confidence limits. ${ }^{29}$ Population estimates were rounded to the nearest 500 persons, per Statistics Canada security regulations. These regulations prevented us from obtaining information on medication prevalence if the number of individuals within a subgroup under study was $<10$; we therefore assigned arbitrary minimum prevalences (1\% for individual medications, $3 \%$ for combinations, see below) to use for those analyses. For details, see Supplemental Methods. For analyses related to 22q11.2DS data we used SAS 9.4 (SAS Institute, Cary, NC, USA) and/or SPSS Version 24 (IBM Corp.). We estimated prevalence of medication use by calculating percentages and Clopper Pearson exact 95\% confidence limits.

Multimorbidity comparisons. We compared the prevalence of multimorbidity for the adult age groups most relevant to 22q11.2DS using those defined in a previous report ${ }^{30}$ of CHMS cycles 1 and 2: 17-24 years, 25-44 years, 45-64 years, 65-69 years, and 70+ years. Given that just two individuals in the 22q11.2DS sample were over age 64 years, we used only population data for the eldest two groups. For comparisons by sex, we used the largest age group in the 22q11.2DS sample, $25-44$ years $(n=127)$. We also determined subsamples on no antipsychotic medications for the largest age groups, 17-24 years $(n=67)$ and $25-44$ years $(n=72)$, given the known high prevalence of psychotic illness in 22q11.2DS. ${ }^{13}$

For all between-group prevalence comparisons, we used the prevalence estimates to calculate prevalence ratios (PRs). We also determined approximations of $95 \%$ CIs for the PR, and when these did not include 1, we reported significance at $p<$ 0.05. For details, see Supplemental Methods.

Medication patterns. To study the pattern of medication use, we examined the prevalence of level 3 ATC classes. ${ }^{28}$ Solely for this purpose we counted individuals on more than one medication in the same class category (e.g., two oral hypoglycemics) just once. We reported all classes at $1 \%$ prevalence or greater, within security regulations. Within the group with multimorbidity we also reported the prevalence of different combinations of three medication classes (triads) at 3\% or greater (instead of $1 \%$, to minimize the time and statistical computing power required to look at every possible combination of three). To compare the pattern of medication use by age group and among those with multimorbidity, we compared medication classes and triads at a prevalence of $5 \%$ or more in either data set. For each between-group comparison, we calculated the PR and 95\% CI approximations for PR, as above.

Predictors of multimorbidity in 22q11.2DS. For the 22q11.2DS sample, we investigated the impact of clinical and demographic variables on multimorbidity. The variables considered were age at medication recording, sex, and clinical variables representing those previously associated with increased medication use ${ }^{23,31}$ and/or major ascertainment features for 22q11.2DS: major congenital cardiac disease (moderate or complex), ${ }^{32}$ presence of ID (moderate or severe), ${ }^{33}$ and presence of psychotic illness (including schizophrenia, schizoaffective disorder, or psychotic depression). ${ }^{34}$ We used multivariate logistic regression to examine the independent effect of predictors on the outcome of 0-4 vs. 5 or more medications. Assumptions of logistic regression were tested to ensure no violations: a quadratic age term was not significant, suggesting no violation of linearity of the logit; Cook's $D$ was $<1$ in all cases, suggesting the absence of influential observations; and variance inflation values did not exceed 2, suggesting no issues with multicollinearity. $P<0.05$ was used to determine significance.

\section{RESULTS}

\section{Prevalence of multimorbidity}

At a median age of 27.8 (Q1 21.7, Q3 39.5) years, the overall prevalence of multimorbidity in 22 q11.2DS was $28.4 \%$ (75/ 264). Figure 1 shows the prevalence of multimorbidity for each age group examined. The prevalence of multimorbidity was significantly higher in 22q11.2DS for the 25-44 year age group compared with the general population aged 25-44 years $(34.7 \%$ vs $2.9 \%, \mathrm{PR}=11.9,95 \% \mathrm{CI} 8.4-17.1)$ and even to those aged $45-64$ years ( $34.7 \%$ vs. $16.4 \%, \mathrm{PR}=2.1,95 \% \mathrm{CI}$ 1.6-2.7). The prevalence of multimorbidity in 25-44 year olds with 22q11.2DS was similar to that in the general population aged $65-69$ years $(34.7 \%$ vs. $32.5 \%, \mathrm{PR}=1.1,95 \% \mathrm{CI} 0.8-1.4)$ (Fig. 1). Restricting to the 22q11.2DS subsample aged 25-44 years on no antipsychotic medications, the prevalence of multimorbidity was also significantly higher than in the comparable population age group $(18.1 \%$ vs. $2.9 \%, \mathrm{PR}=6.2$, 95\% CI 3.6-10.9), and was similar to those aged 45-64 years (18.1\% vs. $16.4 \%, \mathrm{PR}=1.1,95 \% \mathrm{CI} 0.7-1.8$ ) (Fig. 1). Further details, including similar results for male and female subgroups, and other comparisons, including within the 17-24 year age group, are presented in Supplementary Tables S1A, S1B.

\section{Pattern of medication use}

Table 1 shows medication classes in common use (at $5 \%$ or greater prevalence) in either the 22q11.2DS or general population, by age group. Antipsychotics, antiepileptics, antidepressants, thyroid disease medications (e.g., thyroxine), 


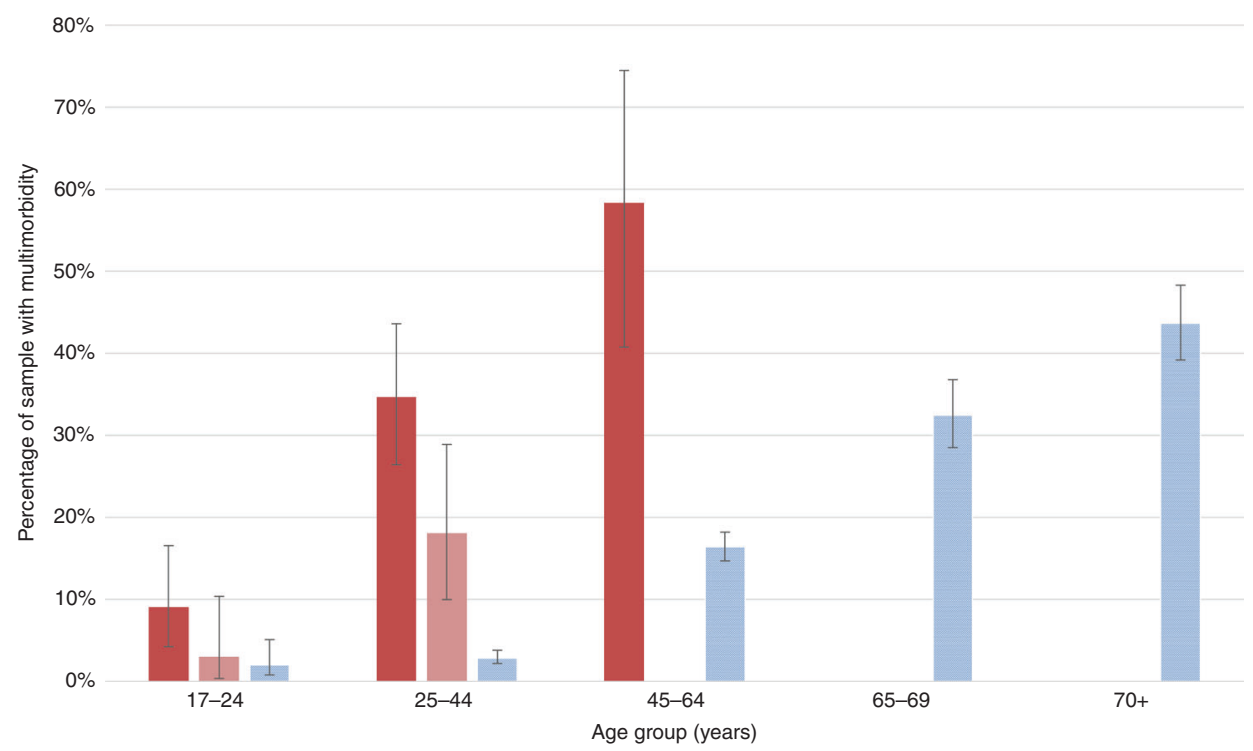

Fig. 1 Multimorbidity (defined as five or more prescription medications) in 22q11.2DS versus the general population, by age group. Plotted are prevalence (\%) and 95\% confidence limits (vertical lines) for each age group in the two samples studied. Solid bars (red) represent the 22q11.2DS sample; lighter shaded bars (pink) represent the subgroup on no antipsychotic medications. Hashed bars (blue) represent the general population sample. Sufficient data for the 65-69 and 70+ year age groups were only available for the general population sample ( $n=2$ and $n=0$, respectively for the 22q11.2DS sample).

and calcitriol were significantly more prevalent in 22q11.2DS, across all age groups (Table 1). Other medication classes $(\beta$ blockers, anticholinergic agents, dopaminergic agents, antithrombotic agents, and corticosteroids) were significantly more prevalent than in the general population only for older age groups (Table 1). Supplementary Tables S2 and S3 provide a complete list of the prevalences of all reportable classes of medication in the 22q11.2DS and population groups.

With respect to medications in common use for those aged 17-24 and/or 25-44 years, there were just two medication classes, antidepressants and hormonal contraceptives, in the general population, but 13 medication classes for the 22q11.2DS sample (Table S2). For the 45-64 year age group, there were ten medications (four from cardiometabolic categories; i.e., medications commonly used to treat heart arrhythmias, hypertension, coronary artery disease, and diabetes) for the general population and 27 for the 22q11.2DS group (including 9 from cardiometabolic categories). Eight of these 27 overlapped the 10 general population common medication classes; for 7 of these 8 , the raw prevalence figures were greater for the 22q11.2DS group (Table S2).

Table 2 shows medication classes in common use among the subgroup with multimorbidity; median age 38.6 (Q1 30.8, Q3 50.2) years for the 22q11.2DS sample, and 62 (Q1 53, Q3 69 ) years for the general population. The 22q11.2DS group with multimorbidity showed significantly higher prevalence of psychotropic medications, thyroid preparations, prescription vitamin D, hormonal contraceptives, and topicals such as corticosteroids and antifungals, but significantly lower prevalence of five cardiometabolic medication classes (Table 2). Further underscoring the difference in the pattern of medication use, the top 5 medication classes among the
22q11.2DS group with multimorbidity were antipsychotics, antidepressants, antiepileptics, thyroid medications, and prescription vitamin $\mathrm{D}$, whereas for the population sample, the top 5 were lipid modifying agents, drugs for gastroesophageal reflux disease, antidepressants, $\beta$-blockers, and angiotensin-converting enzyme (ACE) inhibitors (Table S3); thyroid medications were however in the top 10 .

Table 3 shows combinations of three medication classes, triads, in common use among those with multimorbidity and enriched in the 22q11.2DS group (Table S4 lists all triads reaching a prevalence threshold of $3 \%$ ). The most common triads in the 22q11.2DS group were combinations of neuropsychiatric medications. For example, the combination of an antiepileptic, antipsychotic, and antidepressant, at a prevalence of $28 \%$ was nine times more prevalent in 22q11.2DS than in the general population. Certain endocrinological medications (calcitriol or thyroid preparations) in combination with psychotropic medication, were also significantly more prevalent among the 22q11.2DS group. In contrast, the most common triad in the general Canadian population with multimorbidity, at a prevalence of $8.8 \%$, involved cardiometabolic medications: a $\beta$-blocker, ACE inhibitor, and lipid modifying agent (Table S4).

\section{Predictors of multimorbidity in 22q11.2DS}

Within the 22q11.2DS group, older age and the presence of a psychotic illness, but not sex, major congenital cardiac disease, or ID, were significant predictors of multimorbidity (Table 4).

\section{DISCUSSION}

This study compared the largest available cohort of adults with 22q11.2 deletion syndrome with the general Canadian population. The 22q11.2DS sample with multimorbidity 
Table 1 Prevalence ratios for 29 medication classes in young to middle-aged adults with 22q11.2DS compared with the general population

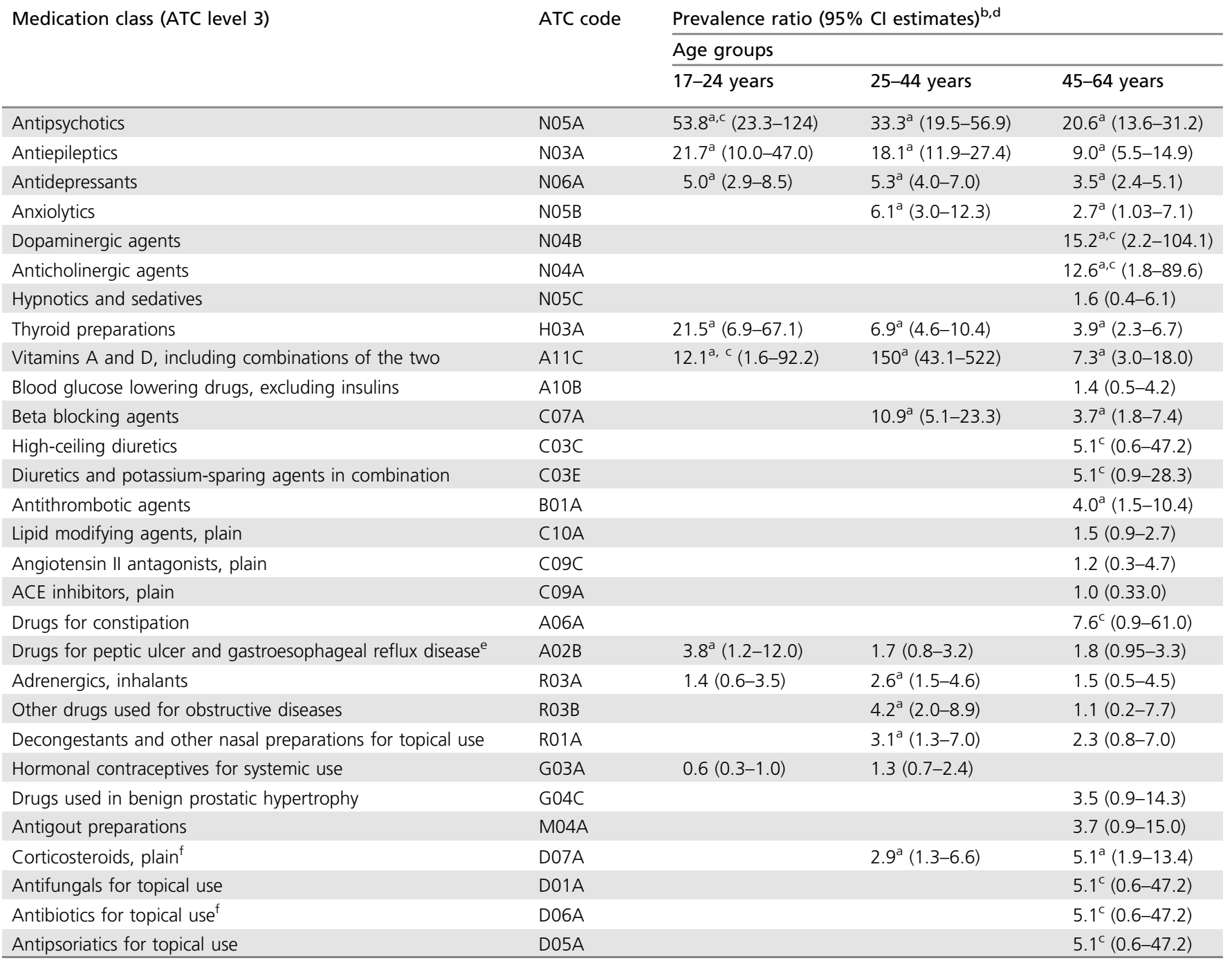

Unfilled boxes represent medications not compared, i.e., raw prevalence $<5 \%$ thus not meeting inclusion criteria for common use.

ACE angiotensin-converting enzyme, ATC Anatomical Therapeutic Chemical Classification System, Cl confidence interval.

aSignificant difference between groups (prevalence ratio and 95\% confidence interval calculations; see "Materials and Methods" and Supplemental Methods for details).

bPrevalence ratios were calculated based on medication use within the age group specified, comparing those in the $22 q 11.2 \mathrm{DS}$ sample $(n=99,127,36$ for age groups

$17-24,25-44,45-65$, respectively) with those in the general population.

'Prevalence too low to be estimated in the Canadian population, given Statistics Canada security regulations. Thus, to calculate the prevalence ratio and estimate $95 \%$ confidence limits, we arbitrarily assigned a 1.0\% value (i.e., lowest possible reportable prevalence) that was reportable in the group (age group or multimorbidity group being compared) and a standard error of $1 \%$. In the $45-64$ year age group, no confidence estimates were reported for $1.0 \%$ prevalence, therefore, we used a $1.1 \%$ prevalence. For more details, see Supplemental Methods.

${ }^{\mathrm{d} C o m p a r i s o n s}$ for three ATC classes (Other Analgesics and Antipyretics [N02B], Anti-inflammatory..., Non-Steroids [M01A], Calcium [A12A]) were not performed as medications in these classes are commonly available over the counter and thus were excluded from the 22q11.2DS sample.

ePrevalences for these drug classes are likely underestimated for the 22q11.2DS group, since any medications that could have been either over the counter and/or noted to be used "as needed" were excluded.

fPrevalence may be overestimated for the 22q11.2DS population, since medications that could have been available over the counter in this class were assumed to be prescription.

(median 38.6 years) was several decades younger on average than the general Canadian population with multimorbidity (median age 62 years). Consistent with our hypothesis, we found that young to middle-aged adults with 22q11.2DS have a greater level of multimorbidity than that of the general population at a comparable age or even several decades older. As a group, 25-44 year olds with 22q11.2DS were most similar in prevalence of multimorbidity to the population sample aged 65-69 years. For the 22q11.2DS subset on no antipsychotic medications, the closest multimorbidity prevalence comparison was with the population aged 45-64 years.

The pattern of medication use in those with multimorbidity also differed between the groups, which was not surprising given their age difference. Those with 22q11.2DS were prescribed more medications used to treat neuropsychiatric 
Table 2 Prevalence ratios for 33 medication classes in the subset of adults with multimorbidity, in 22q11.2DS (median age 38.6 years) compared with the general population (median age 62 years)

Medication class (ATC level 3)

\section{Antipsychotics
Antiepileptics}

Antidepressants

Anticholinergic agents

Dopaminergic agents

Anxiolytics

Hypnotics and sedatives

Opioids $^{f}$

High-ceiling diuretics

Beta blocking agents

Antithrombotic agents

ACE inhibitors, plain

Low-ceiling diuretics, thiazides

Angiotensin II antagonists, plain

Lipid modifying agents, plain

Selective calcium blockers with mainly

vascular effects

Angiotensin II antagonists, combinations

Thyroid preparations

Vitamin $A$ and $D$, including combinations

of the two

Blood glucose lowering drugs, excluding

insulins

Insulins and analogues

Drugs affecting bone structure and

mineralization

Drugs for peptic ulcer and

gastroesophageal reflux disease

Drugs for constipation

Decongestants and other nasal

preparations for topical use

Adrenergics, inhalants

Other drugs for obstructive airway

diseases, inhalants

Hormonal contraceptives for systemic use

Urologicals

Drugs used in benign prostatic hypertrophy

Estrogens

Corticosteroids, plaing

Antifungals for topical use

ACE angiotensin-converting enzyme, ATC Anatomical Therapeutic Chemical Clas-

sification System, $C l$ confidence interval

${ }^{a}$ Significant difference between groups (prevalence ratio and 95\% confidence interval calculations; see "Materials and Methods" and Supplemental Methods for details).

brevalence ratios were calculated based on medication use within the group with multimorbidity at any age (see "Materials and Methods" for details).

'Prevalence too low to be estimated in the Canadian population, given Statistics Canada security regulations. Thus, to calculate the prevalence ratio and estimate $95 \%$ confidence limits, we arbitrarily assigned a $1.0 \%$ value (i.e., lowest possible reportable prevalence) that was reportable in the group (age group or multimorbidity group being compared) and a standard error of $1 \%$. For more details, see Supplemental Methods.

dPrevalence equals 0 for the 22q11.2DS population. For the upper $95 \%$ confidence limit we used the approximation $3 / N^{40}$ Here, $95 \% \mathrm{Cl}$ for the prevalence ratio was not calculable (see Supplemental Methods).

${ }^{\text {e}}$ Comparisons for three ATC classes (Other Analgesics and Antipyretics [N02B], Anti-inflammatory..., Non-Steroids [M01A], Calcium [A12A]) were not performed as medications in these classes are commonly available over the counter and thus were excluded from the 22q11.2DS sample.

fPrevalences for these drug classes are likely underestimated for the 22q11.2DS group, since any medications that could have been either over the counter and/or noted to be used "as needed" were excluded.

grevalence may be overestimated for the 22q11.2DS population, since medications that could have been available over the counter in this class were assumed to be prescription.

and endocrinological conditions than the general population, with the exception of antidepressants. In contrast, the burden of population-based multimorbidity was concentrated in drug categories used to treat cardiovascular/metabolic disease and mood/anxiety disorders, a pattern widely observed in other studies. ${ }^{35}$ In the 22q112DS sample, older age and psychotic illness were significant contributors to multimorbidity. This is consistent with findings reported in previous studies, ${ }^{3}$ suggesting that similar factors contribute to multimorbidity in $22 \mathrm{q} 11.2 \mathrm{DS}$ as in the general population. However, the high level of multimorbidity at a young age in the absence of psychotic illness suggests that other as yet unidentified factors are also important, or that the relationship between contributors and multimorbidity may be differently weighted or shaped in the presence of a 22q11.2 deletion.

Investigation of the pattern of medication use in the multimorbid 22q11.2DS sample led to the identification of several highly prevalent medication class triads, compared with the general population sample. Consistent with findings in other studies, ${ }^{8}$ multimorbidity in the older general population demonstrated relatively higher variability of disease clusters. The genetic homogeneity of the 22q11.2DS sample may have contributed to the patterns observed. In the general population, there may be similar distinct homogeneous subpopulations, each with their own characteristic set of multimorbidity profiles. Some of these may represent individuals with as yet undetected pathogenic CNVs or other rare genetic disorders. Although medication prescribing practices and 22q11.2DS-specific guidelines may also contribute to the homogeneity in medication profiles, ${ }^{27}$ this effect was partially mitigated by not including calcium and vitamin D supplements.

There is a growing recognition of the importance of studying the life course of conditions diagnosed in childhood, such as cerebral palsy, leading to exploration of multimorbidity profiles in young adults. ${ }^{36}$ Although the pattern of diseases in cerebral palsy was quite different to our findings for 22q11.2DS, there was increased prevalence of multimorbidity for those with, compared with those without, cerebral palsy, especially among those with more severe motor impairment. ${ }^{36}$ We note that pathogenic CNVs are major causes of cerebral palsy. ${ }^{37}$

Another group prone to increased multimorbidity at a young age are those diagnosed with ID. The pattern of multimorbidity we found in young adults with 22q11.2DS was somewhat similar to that observed in adults with $\mathrm{ID},{ }^{38}$ where quantitatively, young adults in their 20s were more similar to population controls in their 50s. Consistent with our findings, the odds of neuropsychiatric conditions, especially epilepsy, schizophrenia, and anxiety, were much higher for those with ID, and the odds of cardiovascular conditions were lower. ${ }^{38}$ ID however was not a significant contributor to multimorbidity in our 22q11.2DS sample, even when including any degree of ID (results not shown). Only a minority of those with 22q11.2DS have a formal ID diagnosis. ${ }^{33}$, 39 However, the results suggest that genetic changes leading to even minor cognitive deficits, as in 22q11.2DS, may be associated with a high degree of multimorbidity.

\section{Implications}

We found multimorbidity to be common among young to middle-aged adults with our model genetic disorder, 


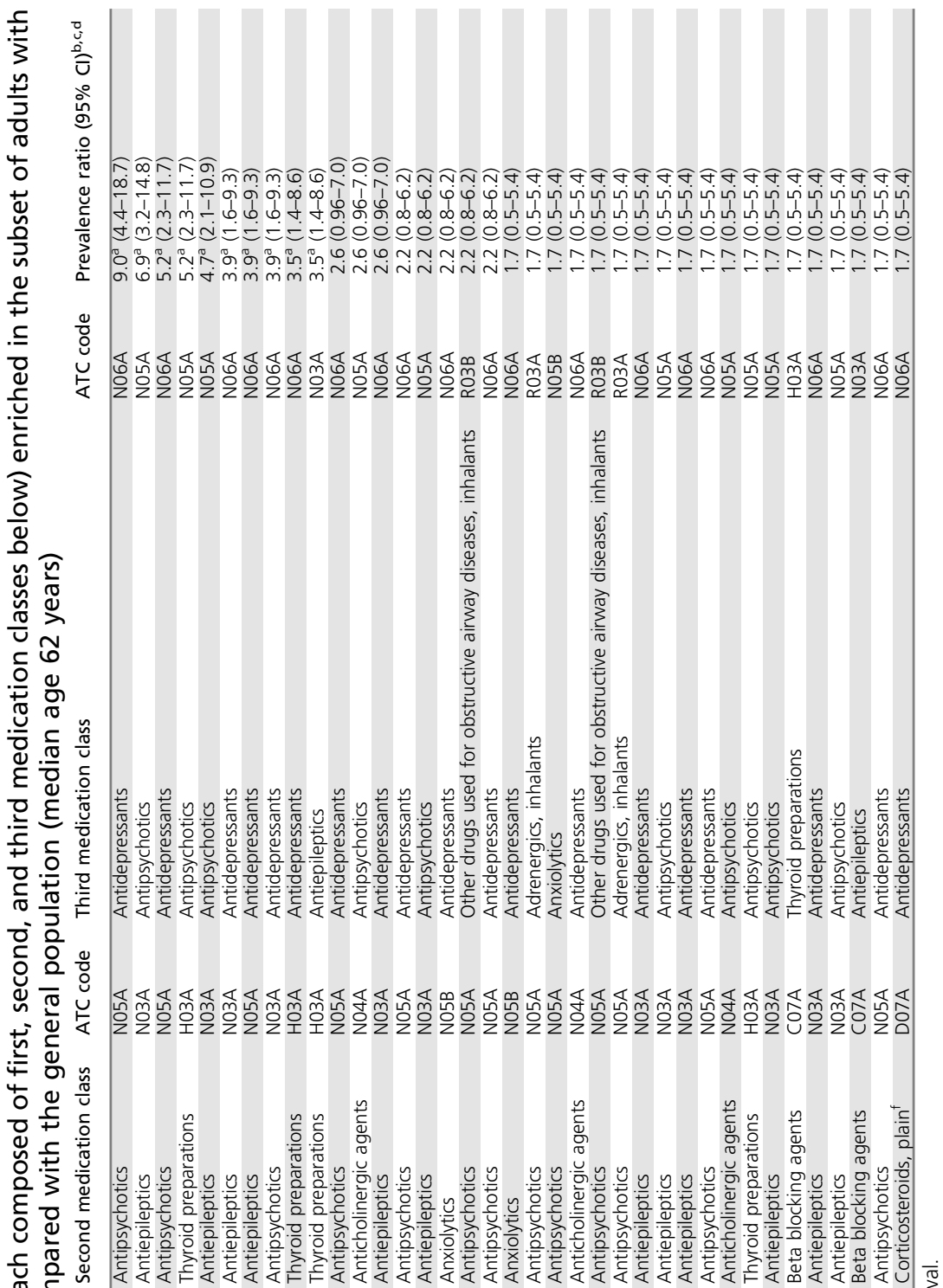

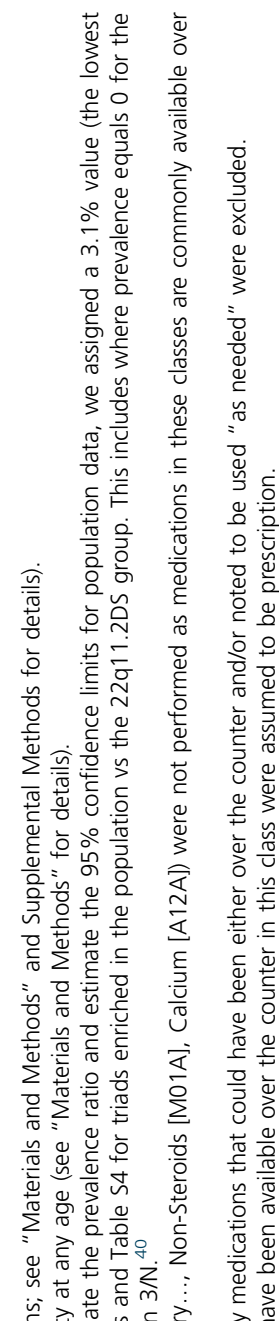

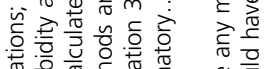

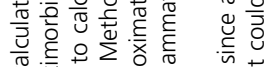

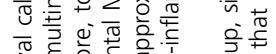
焉 능

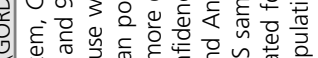

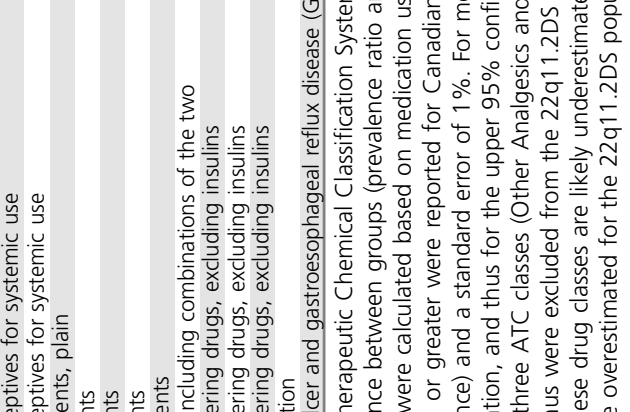
n

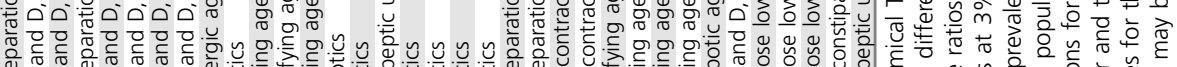

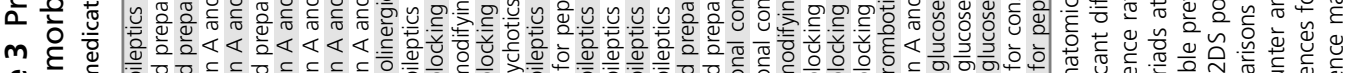

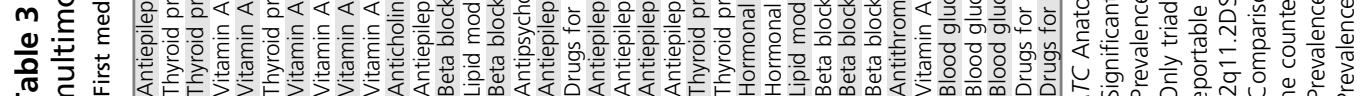


Table 4 Predictors of multimorbidity in adults with 22q11.2DS

Logistic regression analysis

\begin{tabular}{|c|c|c|c|c|c|c|c|c|c|}
\hline \multirow[t]{2}{*}{ Predictor } & \multicolumn{2}{|c|}{ Total sample $N=264$} & \multicolumn{2}{|c|}{ Multimorbidity $N=75$} & \multicolumn{2}{|c|}{ No multimorbidity $N=189$} & \multirow[t]{2}{*}{ OR } & \multirow[t]{2}{*}{ Wald $95 \% \mathrm{Cl}$} & \multirow[t]{2}{*}{$P$} \\
\hline & Median & $(\mathrm{Q} 1,3)$ & Median & $(\mathrm{Q} 1,3)$ & Median & $(\mathrm{Q} 1,3)$ & & & \\
\hline \multirow[t]{2}{*}{ Age (years) } & 27.8 & $(21.7,39.5)$ & 38.6 & $(30.8,50.2)$ & 25.5 & $(20.8,33.1)$ & 1.07 & $1.04-1.10$ & $<0.0001$ \\
\hline & $N$ & $\%$ & $N$ & $\%$ & $N$ & $\%$ & & & \\
\hline Psychotic illness & 109 & 41.3 & 55 & 73.3 & 54 & 28.6 & 5.48 & $2.73-11.00$ & $<0.0001$ \\
\hline ID & 23 & 8.7 & 8 & 10.7 & 15 & 7.9 & 1.70 & $0.55-5.25$ & 0.35 \\
\hline $\mathrm{CHD}$ & 101 & 38.3 & 16 & 21.3 & 85 & 45.0 & 0.79 & $0.37-1.70$ & 0.55 \\
\hline Female sex & 140 & 53.0 & 41 & 54.7 & 99 & 52.4 & 1.10 & $0.58-2.10$ & 0.76 \\
\hline
\end{tabular}

Multimorbidity $=5$ or more medications, no multimorbidity = less than 5 medications. Age is age at medication recording. Q1,3= lower quantile, upper quantile. See "Materials and Methods" for details. Overall the model was significant ( $\left.\chi^{2}[\mathrm{df} 5]=76.3, p<0.0001\right)$. Signifiant $P$ values are bolded.

$C H D$ congenital heart disease (moderate or complex), ${ }^{32} \mathrm{Cl}$ confidence interval, ID intellectual disability (moderate or severe), ${ }^{33}$ OR odds ratio.

22q11.2DS. Coupled with observed increased survival in recent years among individuals with this ${ }^{15}$ and other rare diseases, the findings suggest that such conditions may be contributing to the growing burden of multimorbidity observed in younger adults. Advancements in pediatrics have transformed individually rare, often lethal conditions, into collectively common, chronic, often multimorbid conditions. Individuals with pathogenic CNVs like the recurrent 22q11.2 deletion often have no immediately recognizable pattern of features. ${ }^{13}$ This is in contrast to more familiar genetic conditions like Down syndrome and fragile X syndrome, for which high degrees of multimorbidity at young ages, and/or increased health-care utilization, have previously been reported. ${ }^{9,} 10$ Adults with 22q11.2DS, and the increasing number of other conditions now molecularly diagnosable, are often "hidden in plain sight."

This study represents a first step toward characterizing the pattern of multimorbidity in a model genetic condition with known increased mortality. ${ }^{15}$ Similar characterization of other rare conditions may be helpful for improved coding/ recognition of these conditions in administrative databases, ${ }^{16}$ given that International Classification of Disease (ICD) coding is notoriously poor for congenital defects ${ }^{17}$ and rare diseases. ${ }^{18}$ This is especially the case for conditions increasingly being identified with genetic testing advances, e.g., by gene panels, genome-wide microarrays, or exome/genome sequencing, where specific ICD codes for diseases may not exist. We identified a number of differences in the patterns of medication use between adults with 22q11.2DS and the general population. These patterns may be useful for developing algorithms for data-mining studies to identify adults with 22q11.2DS from large administrative data sets, which could lead to more robust studies with appropriate controls.

Our results highlight the importance of providing interdisciplinary person-centered care for young to middle-aged adults who are at high risk of multimorbidity. To date, there is limited evidence on effective interventions to improve care for people in general with multimorbidity. ${ }^{2}$ A focus on underlying etiology, such as detectable genetic conditions, may be more fruitful than generic approaches.

\section{Strengths and limitations}

To our knowledge, this study is the first to attempt to characterize multimorbidity in adults with a genetic condition, other than Down syndrome, compared with general population data. Future studies are required to validate medication use as a proxy for multimorbidity in the 22q11.2DS population, and confirm its association with health-care utilization in adulthood, and with premature mortality (median age at death 46.4 years). ${ }^{15}$

Using prescription medication as a proxy for multimorbidity has several potential benefits (see "Materials and Methods"). ${ }^{1,19-23}$ However, whether a medication is prescribed depends partially on availability and cultural practices, thus medication prescribing may reflect biases of the prescriber rather than characteristics of the condition itself. ${ }^{1}$ One example would be recommending vitamin D supplements as prophylaxis for hypocalcemia in $22 \mathrm{q} 11.2 \mathrm{DS} .{ }^{27} \mathrm{We}$ mitigated this concern by eliminating supplements from medication counts. However, there may be other less wellrecognized biases for which we have not accounted.

Although we strove for consistency, another limitation of this study is that there may be differences in methodology between prescription drug data for our 22q11.2DS sample and the Canadian sample. Clinical samples, although the only source currently available for studying those with 22q11.2DS, are prone to ascertainment bias, and may result in oversampling of more complex individuals. We examined a subgroup of our sample on no antipsychotics to attempt to mitigate this, but there may be other inherent biases we could not account for, such as those related to data coming from one clinic for the 22q11.2DS sample, where anticipatory care and screening guidelines are followed, ${ }^{27}$ compared with nationally collected population data. Another difference between the two samples was the source of medication data. The 22q11.2DS medication data were derived from medical records and periodic health assessments, whereas the Canadian population data were derived from survey data, which are often prone to self-reporting biases and underreporting. This concern was mitigated by the fact that survey participants' prescription bottles were reviewed prior to data entry. 
For the 22q11.2DS sample, data were limited to the available medical records that did not allow distinguishing between prescription and over-the-counter medications, i.e., some over-the-counter medications may have been prescribed by a physician. We attempted to be conservative by excluding all medications that could potentially be used over the counter or used inconsistently, and comparing this with the maximum number of prescription medications recorded in the Canadian sample (Supplemental Methods). Nevertheless, some assumptions may have been inaccurate. This conservative approach also increased the likelihood that medication use may be underestimated in our 22q11.2DS sample.

\section{Conclusion}

The prevalence of multimorbidity in young to middle-aged adults with 22q11.2DS is comparable with that of the general population several decades older, representing a significant burden of illness. The pattern of multimorbidity tended to be consistent with chronic neuropsychiatric and endocrinological diseases commonly associated with 22q11.2DS. With age, however, medication profiles evolved to become more similar to the cardiometabolic pattern associated with multimorbidity in the far older general population. Further investigations are required to characterize relevant associated health-care costs and relationship to premature mortality in 22q11.2DS. Nonetheless, this study may act as a model for investigating underlying etiologies of the increasing numbers of younger adults with multimorbidity in the general population.

\section{SUPPLEMENTARY INFORMATION}

The online version of this article (https://doi.org/10.1038/s41436019-0603-1) contains supplementary material, which is available to authorized users.

\section{ACKNOWLEDGEMENTS}

The authors thank the adults with 22q11.2DS and their families, as well as the many referring clinicians, for their generous contributions to this and related research studies. The authors also thank the staff at the Toronto Region Statistics Canada Research Data Centre. This work was supported by the Canadian Institutes of Health Research (A.S.B., MOP \#97800, MOP \#111238), Dalglish Chair (A.S.B.) and Dalglish Fellow (E.Boot) awards, Canada Research Chairs program (A.S.B.), Mr. Rajeeb Mukherjee BSc (Hons) (London School of Economics) Fellowship in Schizophrenia (E.Breetvelt), a McLaughlin Centre Accelerator Grant, and National Institute of Mental Health grant U01 MH101723-01(3/5). The Dalglish Family 22q Clinic was founded by a grant from the W. Garfield Weston Foundation.

\section{DISCLOSURE}

The authors declare no conflicts of interest.
Publisher's note: Springer Nature remains neutral with regard to jurisdictional claims in published maps and institutional affiliations.

\section{REFERENCES}

1. Bastian LA, Brandt CA, Justice AC. Measuring multimorbidity: a risky business. J Gen Intern Med. 2017;32:959-960.

2. Smith SM, Soubhi H, Fortin M, Hudon C, O'Dowd T. Managing patients with multimorbidity: systematic review of interventions in primary care and community settings. BMJ. 2012;345:e5205.

3. Barnett K, Mercer SW, Norbury M, Watt G, Wyke S, Guthrie B. Epidemiology of multimorbidity and implications for health care, research, and medical education: a cross-sectional study. Lancet. 2012;380:37-43.

4. Gijsen R, Hoeymans N, Schellevis FG, Ruwaard D, Satariano WA, van den Bos GA. Causes and consequences of comorbidity: a review. J Clin Epidemiol. 2001;54:661-674.

5. The Academy of Medical Sciences. Multimorbidity: a priority for global health research. 2018. https://acmedsci.ac.uk/policy/policy-projects/ multimorbidity. Accessed 10 November 2018.

6. Hawkes N. Treatment for those with multiple serious illnesses is "ineffective," warns major review. BMJ. 2018:361:k1736.

7. Lancet T. Making more of multimorbidity: an emerging priority. Lancet. 2018;391:1637.

8. Pefoyo AJ, Bronskill SE, Gruneir A, et al. The increasing burden and complexity of multimorbidity. BMC Public Health. 2015;15:415.

9. Alexander M, Petri H, Ding Y, Wandel C, Khwaja O, Foskett N. Morbidity and medication in a large population of individuals with Down syndrome compared to the general population. Dev Med Child Neurol. 2016;58:246-254.

10. Nazareth T, Li N, Marynchenko M, et al. Burden of illness among patients with fragile $X$ syndrome (FXS): a Medicaid perspective. Curr Med Res Opin. 2016;32:405-416.

11. Gravholt CH, Juul S, Naeraa RW, Hansen J. Morbidity in Turner syndrome. J Clin Epidemiol. 1998;51:147-158.

12. Bassett AS, Chow EW, Husted J, et al. Clinical features of 78 adults with 22q11 Deletion syndrome. Am J Med Genet A. 2005;138:307-313.

13. McDonald-McGinn DM, Sullivan KE, Marino B, et al. 22q11.2 deletion syndrome. Nat Rev Dis Primers. 2015;1:15071.

14. Roubertoux PL, de Vries PJ. From molecules to behavior: lessons from the study of rare genetic disorders. Behav Genet. 2011;41:341-348.

15. Van L, Heung T, Graffi J, Ng E, Malecki SL, Van Mil S, Boot E, et al. Allcause mortality and survival in adults with 22q11.2 deletion syndrome. Genet Med. 2019 Apr 5; https://doi.org/10.1038/s41436-019-0509-y [Epub ahead of print].

16. Nielsen $E H$, Lindholm J, Laurberg $P$. Use of combined search criteria improved validity of rare disease (craniopharyngioma) diagnosis in a national registry. J Clin Epidemiol. 2011;64:1118-1126.

17. Holmes LB, Westgate MN. Using ICD-9 codes to establish prevalence of malformations in newborn infants. Birth Defects Res A Clin Mol Teratol. 2012;94:208-214.

18. Ayme S, Bellet B, Rath A. Rare diseases in ICD11: making rare diseases visible in health information systems through appropriate coding. Orphanet J Rare Dis. 2015;10:35.

19. Brilleman SL, Salisbury C. Comparing measures of multimorbidity to predict outcomes in primary care: a cross sectional study. Fam Pract. 2013;30:172-178.

20. Charlson ME, Pompei P, Ales KL, MacKenzie CR. A new method of classifying prognostic comorbidity in longitudinal studies: development and validation. J Chronic Dis. 1987;40:373-383.

21. Violan C, Foguet-Boreu Q, Flores-Mateo G, et al. Prevalence, determinants and patterns of multimorbidity in primary care: a systematic review of observational studies. PLOS ONE. 2014;9:e102149.

22. George J, Vuong T, Bailey MJ, Kong DC, Marriott JL, Stewart K. Development and validation of the medication-based disease burden index. Ann Pharmacother. 2006;40:645-650.

23. O'Dwyer M, Peklar J, McCallion P, McCarron M, Henman MC. Factors associated with polypharmacy and excessive polypharmacy in older people with intellectual disability differ from the general population: a cross-sectional observational nationwide study. BMJ Open. 2016;6: e010505.

24. Harrison C, Britt H, Miller G, Henderson J. Examining different measures of multimorbidity, using a large prospective cross-sectional study in Australian general practice. BMJ Open. 2014;4:e004694. 
25. Voll SL, Boot E, Butcher NJ, et al. Obesity in adults with 22q11.2 deletion syndrome. Genet Med. 2017;19:204-208.

26. Government of Canada. Prescription drug list. 2018. https://www. canada.ca/en/health-canada/services/drugs-health-products/drugproducts/prescription-drug-list/list.html. Accessed 8 June 2018.

27. Fung WL, Butcher NJ, Costain G, et al. Practical guidelines for managing adults with 22q11.2 deletion syndrome. Genet Med. 2015;17: 599-609.

28. WHO Collaborating Centre for Drug Statistics Methodology. ATC/DDD Index 2018. https://www.whocc.no/atc_ddd_index/. Accessed 11 May 2018.

29. Rust KF, Rao JN. Variance estimation for complex surveys using replication techniques. Stat Methods Med Res. 1996;5:283-310.

30. Rotermann M, Sanmartin C, Hennessy D, Arthur M. Prescription medication use by Canadians aged 6 to 79. 2014. https://www. statcan.gc.ca/pub/82-003-x/2014006/article/14032-eng.htm. Accessed 11 May 2018

31. Zink M, Englisch S, Meyer-Lindenberg A. Polypharmacy in schizophrenia. Curr Opin Psychiatry. 2010;23:103-111.

32. Billett J, Cowie MR, Gatzoulis MA, Vonder Muhll IF, Majeed A. Comorbidity, healthcare utilisation and process of care measures in patients with congenital heart disease in the UK: cross-sectional, population-based study with case-control analysis. Heart. 2008;94:1194-1199.
33. Chow EW, Watson M, Young DA, Bassett AS. Neurocognitive profile in 22 q11 deletion syndrome and schizophrenia. Schizophr Res. 2006;87:270-278.

34. Bassett AS, Chow EW, AbdelMalik P, Gheorghiu M, Husted J, Weksberg R. The schizophrenia phenotype in 22q11 deletion syndrome. Am J Psychiatry. 2003;160:1580-1586.

35. Ryan BL, Bray Jenkyn K, Shariff SZ, et al. Beyond the grey tsunami: a cross-sectional population-based study of multimorbidity in Ontario. Can J Public Health. 2018;109:845-854.

36. Whitney DG, Hurvitz EA, Ryan JM, et al. Noncommunicable disease and multimorbidity in young adults with cerebral palsy. Clin Epidemiol. 2018:10:511-519.

37. Oskoui M, Gazzellone MJ, Thiruvahindrapuram B, et al. Clinically relevant copy number variations detected in cerebral palsy. Nat Commun. 2015;6:7949.

38. Cooper SA, McLean G, Guthrie B, et al. Multiple physical and mental health comorbidity in adults with intellectual disabilities: populationbased cross-sectional analysis. BMC Fam Pract. 2015;16:110.

39. Swillen A, McDonald-McGinn D. Developmental trajectories in 22q11.2 deletion syndrome. Am J Med Genet C Semin Med Genet. 2015;169. 172-181.

40. Newman TB. If almost nothing goes wrong, is almost everything all right? Interpreting small numerators. JAMA. 1995;274:1013. 